Check for updates

Cite this: Phys. Chem. Chem. Phys., 2017, 19, 12441

Received 9th February 2017, Accepted 24th April 2017

DOI: $10.1039 / c 7 c p 00877 e$

rsc.li/pccp

\title{
Tuning crystalline ordering by annealing and additives to study its effect on exciton diffusion in a polyalkylthiophene copolymer $\dagger$
}

\author{
Mithun Chowdhury, (D) $\ddagger \S^{a}$ Muhammad T. Sajjad, $\ddagger^{a}$ Victoria Savikhin, bd \\ Noémie Hergué, ${ }^{c}$ Karina B. Sutija, ${ }^{a}$ Stefan D. Oosterhout, ${ }^{b}$ Michael F. Toney, (D) ${ }^{b}$ \\ Philippe Dubois, $\mathbf{9}^{c}$ Arvydas Ruseckas ${ }^{a}$ and Ifor D. W. Samuel (D) $\star^{a}$
}

\begin{abstract}
The influence of various processing conditions on the singlet exciton diffusion is explored in films of a conjugated random copolymer poly-(3-hexylthiophene-co-3-dodecylthiophene) (P3HT-CO-P3DDT) and correlated with the degree of crystallinity probed by grazing incidence $\mathrm{X}$-ray scattering and with exciton bandwidth determined from absorption spectra. The exciton diffusion coefficient is deduced from exciton-exciton annihilation measurements and is found to increase by more than a factor of three when thin films are annealed using $\mathrm{CS}_{2}$ solvent vapour. A doubling of exciton diffusion coefficient is observed upon melt annealing at $200{ }^{\circ} \mathrm{C}$ and the corresponding films show about $50 \%$ enhancement in the degree of crystallinity. In contrast, films fabricated from polymer solutions containing a small amount of either solvent additive or nucleating agent show a decrease in exciton diffusion coefficient possibly due to formation of traps for excitons. Our results suggest that the enhancement of exciton diffusivity occurs because of increased crystallinity of alkyl-stacking and longer conjugation of aggregated chains which reduces the exciton bandwidth.
\end{abstract}

\section{Introduction}

Semiconducting polymers are widely studied due to their ease of processing and use in high performance optoelectronic devices such as organic photovoltaic cells (OPVs). ${ }^{1,2}$ In OPVs, dissociation of excitons at the interface between donor and acceptor is one of the important processes that determines the photoconversion efficiency. The short exciton diffusion length

\footnotetext{
${ }^{a}$ Organic Semiconductor Centre, SUPA, School of Physics and Astronomy, University of St. Andrews, North Haugh, St. Andrews, KY16 9SS, UK. E-mail: idws@st-andrews.ac.uk

${ }^{b}$ Stanford Synchrotron Radiation Lightsource, Menlo Park, CA 94025, USA

${ }^{c}$ Laboratory of Polymeric and Composite Materials, University de Mons, Mons, Belgium

${ }^{d}$ Stanford Electrical Engineering Department, Stanford, CA 94305, USA

$\dagger$ Electronic supplementary information (ESI) available: GIXS analysis to determine relative degrees of crystallinity, comparison between P3DDT and P3HT-coP3DDT, fluorescence decays measured at different excitation densities, exciton diffusion length, dependence of exciton diffusion coefficient on crystallite size, AFM height topography, synthesis, DSC thermogram and NMR data, calculation of free exciton bandwidth from absorption spectra of aggregated chains. See DOI: $10.1039 / \mathrm{c} 7$ cp00877e

\$ M. C. and M. T. S. contributed equally to this work.

$\S$ Present address: Department of Chemical and Biological Engineering, Princeton University, Princeton, NJ 08544, USA.

T Present address: MRT Department, Luxembourg Institute of Science and Technology - LIST, Belvaux, Luxembourg.
}

is an important factor that limits the number of excitons that can reach the interface. A significant amount of work has been done to optimize the morphology of the active layer in the blend in order to reduce the distance between exciton generation and interface, ${ }^{3,4}$ but less attention has been paid to increasing exciton diffusion. A few attempts have been made to enhance the exciton diffusion length using phosphorescent dopant, ${ }^{5}$ wide bandgap host, ${ }^{6}$ thermal annealing ${ }^{7}$ and single crystals ${ }^{8}$ or highly crystalline small molecules, ${ }^{6,9,10}$ however enhancing exciton diffusion in semi-crystalline conjugated polymers ${ }^{11,12}$ and correlating it to tunable intermolecular interactions, morphology and crystalline order are not so well explored even in the well-studied poly(3-hexylthiophenes) (P3HT). ${ }^{13,14}$ Recently air-stable solar cells with power conversion efficiencies of $6.4 \%$ have been demonstrated using blends of P3HT with new electron acceptors which were thermally annealed at $130{ }^{\circ} \mathrm{C} .{ }^{15}$ But it has also been shown for small molecules that processing methods (such as using solvent additive and thermal annealing) can decrease the exciton diffusion length which has been explained by formation of exciton traps. ${ }^{10,16}$

In order to establish structure-property relations for exciton diffusion in semi-crystalline conjugated polymers, it is instructive to control intermolecular interactions and so control film morphology and crystallinity. A range of processing protocols such as using solvent additives, ${ }^{4,17-19}$ polymer crystal nucleating agents, ${ }^{20,21}$ 
thermal $^{7}$ and solvent vapour annealing ${ }^{22}$ have been used to control crystallisation in conjugated polymers, inspired from the protocols of classical semi-flexible polymers. ${ }^{23,24}$ Out of these, appropriate annealing is a simple and useful method which enables the arrangement of polymer chains to change in a controllable fashion. ${ }^{7,22,25,26}$ There is a report of thermal annealing improving exciton diffusion in semi-crystalline conjugated polymers, ${ }^{7}$ however, other studies reported a decrease or no change. ${ }^{10,27}$ As semiconducting conjugated polymers consist of long chains, there are several other factors such as entanglement, lamellar orientation, and interchain interactions, that could govern the molecular level ordering or crystallinity. ${ }^{28,29}$

Here we show that thermal, melt, and solvent vapour annealing can lead to extended chains of oriented lamellae and enhanced degrees of crystallinity in spin-coated thin films of the copolymer poly(3-hexylthiophene-co-3-dodecylthiophene) (P3HT-co-P3DDT). We found that the exciton diffusion coefficient increases with increasing crystallinity of the copolymer upon annealing. The most pronounced changes in exciton diffusion coefficient were on melt-annealed films for which a doubling was observed, and for solvent (carbon disulphide) vapour annealed films for which a $\sim 3$-fold enhancement was observed. To understand the additional role of processing additives, we also investigated thermal and melt annealing of films made with small amounts of additives. In these films thermal and melt annealing increase the degree of crystallinity, but the exciton diffusion coefficient decreases. We attribute this to the additives leading to the formation of traps. This work shows how processing techniques can lead to large increases in exciton diffusion and relates the changes to crystallinity.

\section{Results}

We explored the polyalkylthiophene copolymer P3HT-co-P3DDT $\left(M_{\mathrm{n}}=34 \mathrm{kD}, \mathrm{PDI}=1.19\right.$, hexyl $:$ dodecyl $\sim 53: 47$, regioregularity $\sim 99 \%$ (i.e., extent of head-to-tail coupling of thiophene units, see Fig. 1d), melting temperature $=188{ }^{\circ} \mathrm{C}$, crystallisation temperature $=157^{\circ} \mathrm{C}$ ). The polymer has a random arrangement of P3HT and P3DDT sub-units but the directionality of the units is highly regioregular. It is an ideal system to explore in terms of solution processability, regioregularity and semi-crystallinity. It has long polymer chains (molecular weight $M_{\mathrm{n}}=34 \mathrm{kD}$ ), about twice larger than the entanglement molecular threshold weight of $\sim 15 \mathrm{kD}$ needed to form a two-phase amorphous-crystalline morphology leading to partially folded lamellar structures (see Fig. $1 \mathrm{a}-\mathrm{c}){ }^{28,30}$ It has a lower melting point than P3HT which is helpful for applying temperature protocols for annealing, without much worry of degradation. We applied three different processing protocols; thermal annealing $\left(T_{\text {ca }}\right)$, melt annealing ( $\left.T_{\text {ma }}\right)$ and solvent vapour annealing (SvA), to explore their effect on the organisation of the polymer chains. For thermal annealing, we annealed the films a few degrees above the crystallisation temperature $\left(T_{\text {ca }}=160{ }^{\circ} \mathrm{C}\right)$. For melt annealing, the films were annealed a few degrees above the melting point $\left(T_{\mathrm{ma}}=200{ }^{\circ} \mathrm{C}\right)$. In both cases, heating was followed by slow controlled cooling towards room temperature to ensure that the crystallinity stayed close to the thermodynamic equilibrium. Solvent vapour annealing of thin films was done by controlling the flow of carbon disulphide $\left(\mathrm{CS}_{2}\right)$ vapours into a custom-built solvent vapour annealing chamber. The chamber has a Peltier element to control the sample temperature and the amount of solvent which could condense onto a cold film. Further details about the synthesis, processing protocols and characterisation of the copolymer P3HT-co-P3DDT can be found in the Experimental section and ESI. $\dagger$

\subsection{Exciton diffusion and structural measurements of P3HT-co-P3DDT}

To determine the singlet exciton diffusivity we studied singletsinglet exciton annihilation by measuring time-resolved fluorescence at different excitation intensities. Exciton-exciton annihilation occurs at high exciton densities when two excitons interact with each other and one exciton transfers its energy to the other exciton which is promoted to a higher energy state and then usually relaxes back to the lowest energy excited state. As a result, one exciton is lost per encounter. ${ }^{31,32}$ Fluorescence decays measured at different excitation densities are shown in ESI $\dagger$ (Fig. S3). The decays are faster at higher excitation densities because excitons are closer to each other and annihilate faster. Time-resolved fluorescence intensity is proportional to the density of singlet excitons $N$ and can be described by the rate equation:

$$
\frac{\mathrm{d} N}{\mathrm{~d} t}=G-k N-\gamma N^{2}
$$

where $G$ is a generation term, $k$ is the decay rate constant in the absence of annihilation and $\gamma(t)$ is the annihilation rate constant. Because the excitation pulse is very short (200 fs), generation is instantaneous and the PL rises with the instrument response $\left(\sim 5 \mathrm{ps}\right.$ in this case). At very low excitation density $\left(<10^{15} \mathrm{~cm}^{-3}\right)$ the probability for two excitons to encounter each other during their lifetime is very low, hence $k N \gg \gamma N^{2}$ and the decay can be described as $\mathrm{d} N / \mathrm{d} t=-k N$. Nevertheless, the PL decays are nonexponential even at very low excitation density. We used a sum of three exponential functions to describe this decay and to obtain the empirical time-dependent $k(t)$. The rate constant for diffusion-limited annihilation in a three-dimensional isotropic system can be described by a sum of a time-independent term and of a time-dependent term ${ }^{32,33}$

$$
\gamma(t)=4 \pi R_{\mathrm{a}} D\left(1+\frac{R_{\mathrm{a}}}{\sqrt{2 \pi D t}}\right)
$$

where $D$ is the exciton diffusion coefficient and $R_{\mathrm{a}}$ is the annihilation radius, which is the exciton separation radius at which annihilation occurs. Eqn (2) assumes that only one exciton is lost per encounter. The value of $R_{\mathrm{a}}$ was experimentally determined in P3HT to be $1.8 \mathrm{~nm}^{31,32}$ and we use this value for the copolymer studied here because it shows similar packing to $\mathrm{P} 3 \mathrm{HT}$ as observed by Grazing Incidence X-ray scattering (GIXS). ${ }^{30}$ The magnitude of $R_{\mathrm{a}}$ depends on the spectral overlap of fluorescence with excited state absorption which is unlikely 

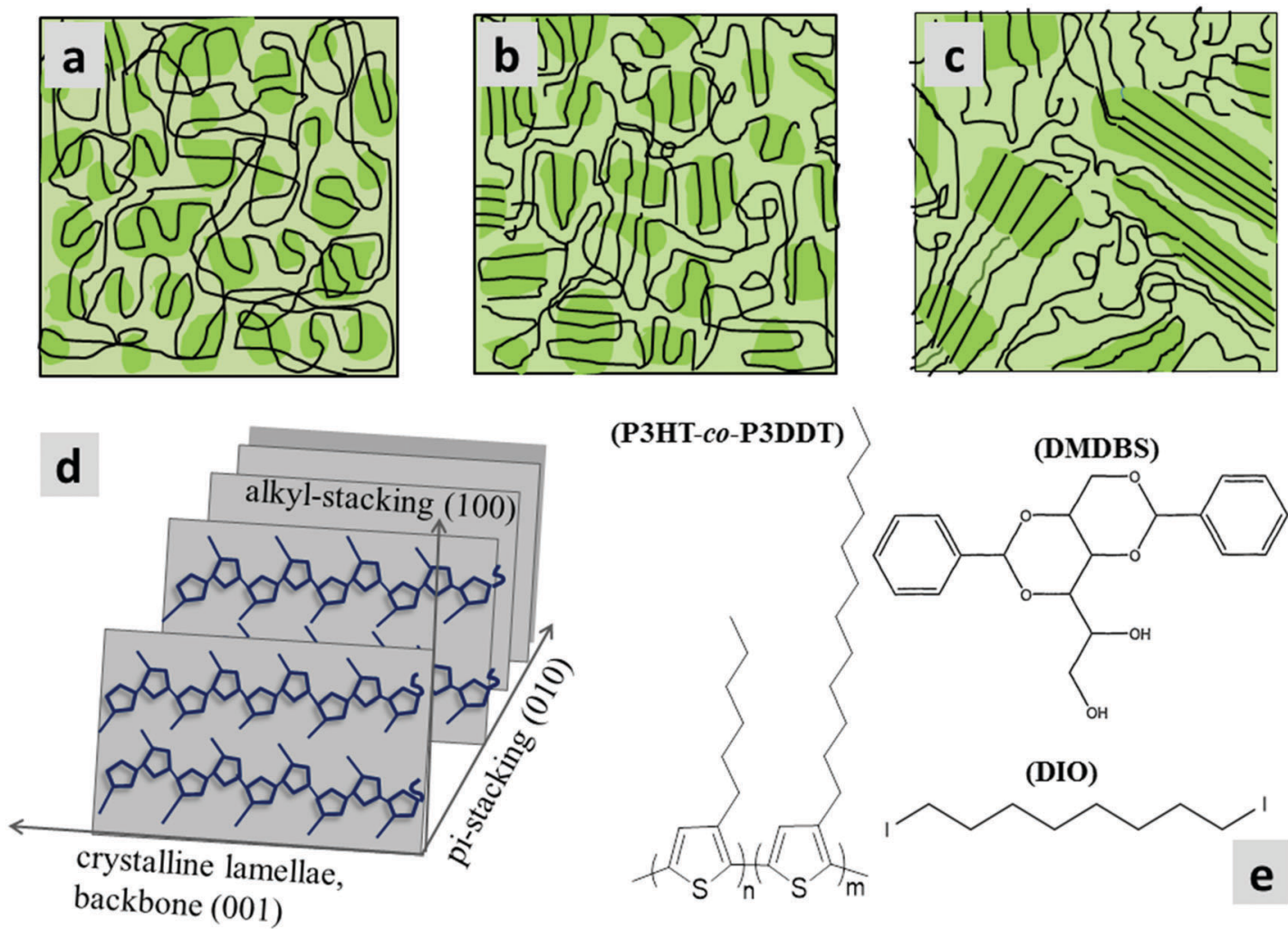

Fig. 1 (a-c) Schematics of microstructures of a semi-crystalline polymer (e.g., P3HT-co-P3DDT) film while at different extent of expected ordered (crystalline)/disordered (amorphous) states, (a) as spin-coated, (b) thermally annealed, (c) melt annealed. Darker shadowed areas represent relatively more ordered portions of the chain as folded lamellae, whereas, amorphous portions (lighter shading) resemble unfolded cooked spaghetti. Co-existence of such folded and unfolded chain microstructure is known as fringed-micellar morphology. (d) Schematic of packing of polyalkylthiophene showing directions of pi-stacking and alkyl-stacking. (e) Chemical structures of the alkylthiophene copolymer (P3HT-co-P3DDT) and DIO, DMDBS additives used in this study.

to change significantly with different processing because changes in ground state absorption and fluorescence spectra are small (vide infra). Hence we assumed that $R_{\mathrm{a}}$ did not change with different processing. We substituted eqn (2) into eqn (1) to simulate the $N(t)$ decay at different excitation densities $\left(>10^{17} \mathrm{~cm}^{-3}\right)$. Simulated $N(t)$ was convolved with the instrument response function to fit the measured fluorescence decays and the fits with one value of $D$ for each sample are shown in Fig. S3 (ESI $\dagger)$. The time-dependent $\gamma(t)$ for different samples is given in Fig. 2. At long times after excitation $(t>100 \mathrm{ps}) \gamma$ is dominated by a time-independent term which indicates that annihilation is controlled by three-dimensional exciton diffusion. Similar behavior has been observed in P3HT films. ${ }^{31,32}$ We determined one-dimensional exciton diffusion length using

$$
L_{D}=\sqrt{D \tau}
$$

where $\tau$ is the decay time of photoluminescence to $1 / e$ of its initial value at very low excitation density of $<10^{15} \mathrm{~cm}^{-3}$ which approximately corresponds to 1 sun illumination conditions and are shown in Fig. S4 (ESI $\dagger$ ). For most films the estimated exciton diffusion length was between 4 and $7 \mathrm{~nm}$ but the solvent-vapour annealed film had a higher diffusion length of $9.5 \pm 1 \mathrm{~nm}$ (Fig. S4 in ESI $\dagger$ ).
We explored the surface morphology and degree of crystallinity of co-polymer films using Atomic Force Microscopy (AFM) and GIXS. An AFM phase image of as-cast film shows folded lamellar structure of polymer chains (Fig. 3a) which resembles packing similar to schematic in Fig. 1a. Fig. 4a shows the 2D area image of GIXS patterns of the as spin-coated film. The Bragg peaks at $q=0.289 \AA^{-1}$ and $q=0.578 \AA^{-1}$ show similar positions to these previously observed in $\mathrm{P} 3 \mathrm{HT}$ films and are attributed to the (100) and (200) crystalline planes defined by stacking of the alkyl chains. ${ }^{34,35}$ They are stronger in the 'out-of-plane' $q_{z}$ direction indicating copolymer chains are predominantly 'edge-on' oriented on the substrate. Bragg peak at $q=1.69 \AA^{-1}$ is attributed to the (010) $\pi-\pi$ stacking and is stronger for the 'in-plane' ' $q_{x y}$ ', direction. The relative degree of crystallinity $(\mathrm{rDoC})^{34}$ is determined by integrating background-subtracted scattering intensity of the (100) and (200) Bragg peaks (respectively at $q_{z}=0.289 \AA^{-1}$ and $q_{z}=0.578 \AA^{-1}$ ) within $q= \pm 0.1 \AA^{-1}$ and $\chi$ range from $2.5^{\circ}$ to $177.5^{\circ}$ as explained in Fig. S1a and $b$ (ESI $\left.\dagger\right)$. The integrated intensities are then normalised by film thickness, exposure time and irradiation length of the sample. For comparison of crystallinity among all the differently processed samples, each rDoC value was normalised by the highest rDoC value in the whole data set, which in our case is the melt-annealed film of the neat polymer. The rDoC values for both (100) and (200) peaks are 

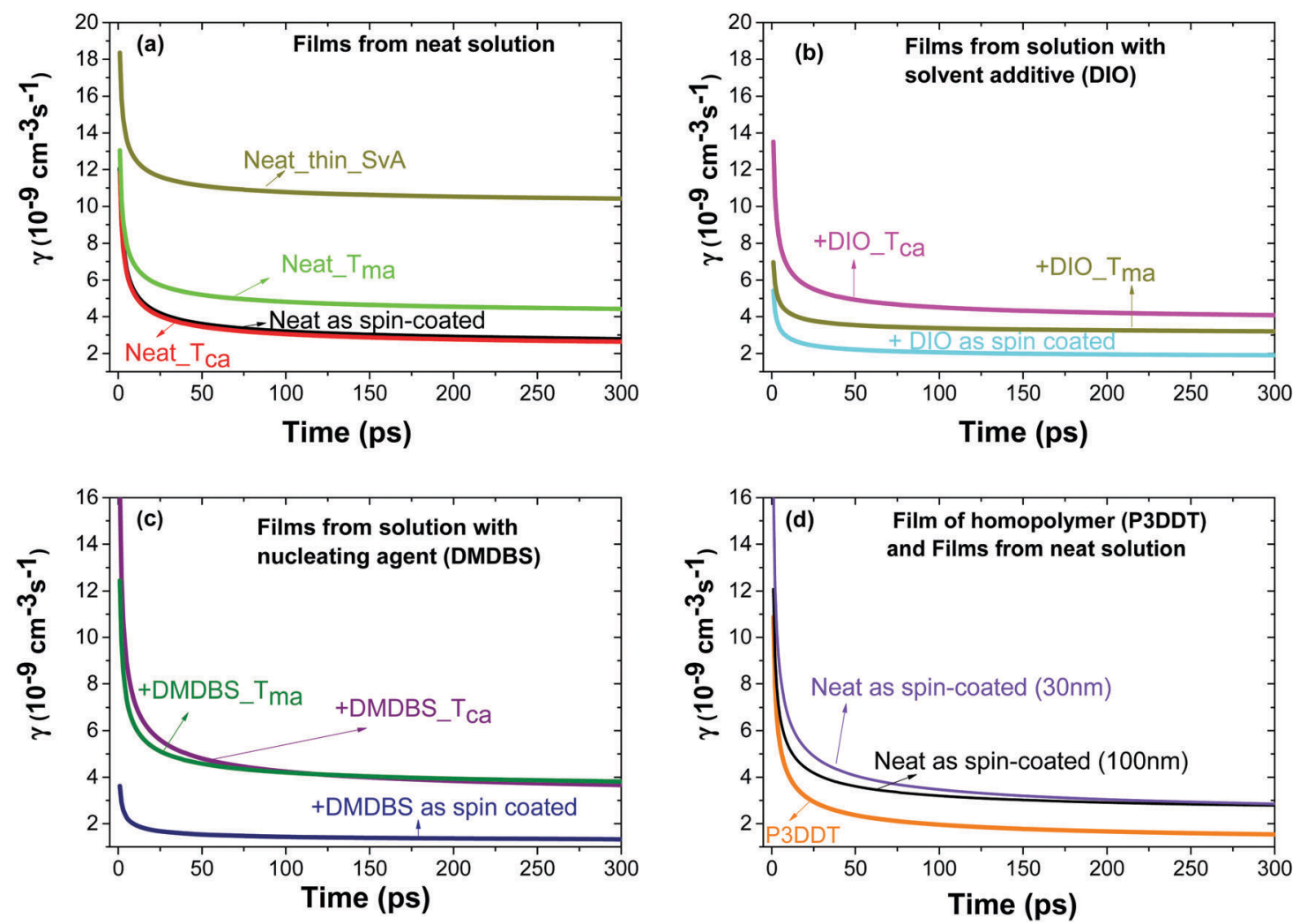

Fig. 2 Rate constant $\gamma$ of singlet-singlet exciton annihilation in films of P3HT-co-P3DDT vs. time after excitation obtained by fitting the fluorescence decays measured at different excitation densities using eqn (1). Films were spin-coated from neat solution in chlorobenzene (a) or with additives DIO (b) or DMDBS (c). Some films where thermally annealed at $T_{\text {ca }}=160{ }^{\circ} \mathrm{C}$, melt annealed at $T_{\text {ma }}=200{ }^{\circ} \mathrm{C}$ or annealed in $\mathrm{CS}_{2}$ vapour (SvA). Panel (d) shows the results for different thicknesses of P3HT-CO-P3DDT films and for a $100 \mathrm{~nm}$ P3DDT film.

nearly the same which gives us confidence in our analysis. We estimate errors of $10 \%$ in rDoC as a result of the film thickness measurement error and experimental calibration error. ${ }^{36,37}$ The crystallite size along the (h00) direction was calculated by considering the width of all (h00) peaks using Williamson-Hall analysis (details in ESI $\dagger$ ). All results are summarised in Table 1.

Separately we measured exciton diffusivity in spin-coated films of homopolymer P3DDT of comparable molecular weight (27 kD) and found $D=0.6 \times 10^{-3} \mathrm{~cm}^{2} \mathrm{~s}^{-1}$ which is twice lower than in the copolymer P3HT-co-P3DDT. GIXS studies and absorption spectra given in Fig. S2b-d (ESI $\dagger$ ) suggest higher ordering in the copolymer as compared to the homopolymer. This observation is in line with prior reports, which show that increasing the length of the side alkyl chain to more than six carbon atoms deteriorates crystallinity and photovoltaic performance. ${ }^{38,39}$ The higher molecular level ordering of our alkylthiophene copolymers is consistent with the reports of enhanced mobility and device performance. ${ }^{40,41}$ This further signifies the importance of characterising the molecular level ordering and crystallinity of copolymer films, which could be altered during various processing methods, and may be responsible for the extent of exciton diffusion. Furthermore, the exciton diffusion coefficient value found for an as spin-coated film of neat P3HT-co-P3DDT $\left(D=1.2 \times 10^{-3} \mathrm{~cm}^{2} \mathrm{~s}^{-1}\right)$ is slightly smaller than the value of $1.8 \times 10^{-3} \mathrm{~cm}^{2} \mathrm{~s}^{-1}$ reported for P3HT films. ${ }^{31}$ This is not surprising because some of the side chains of the copolymer in this study are longer.

\subsection{Influence of thermal and melt annealing}

Spin-coated $\sim 100 \mathrm{~nm}$ thick films of P3HT-co-P3DDT were subjected to annealing protocols around crystallisation $\left(T_{\mathrm{ca}}\right)$ and melting temperature ( $T_{\mathrm{ma}}$ ) (details are given in Experimental section). We measured exciton diffusion coefficient using the procedure described in Section 2.1 and found that it did not change upon thermal annealing but increased from $1.2 \times 10^{-3} \mathrm{~cm}^{2} \mathrm{~s}^{-1}$ to $2.1 \times 10^{-3} \mathrm{~cm}^{2} \mathrm{~s}^{-1}$ upon melt annealing.

AFM phase images shown in Fig. 3 represent spatial variations in the elastic modulus of the material. ${ }^{23,26}$ Stiffer crystalline regions are brighter whilst soft amorphous regions are dark. The image of as spin-coated film from neat solution shows smaller crystalline regions (Fig. 3a), whilst thermal and melt annealing result in a significant increase of the length and width of crystalline domains. This can be explained by larger scale chain rearrangements upon melt annealing and crystallisation of slowly cooled polymer melt. To get more insight into the crystallinity changes we have performed Grazing Incidence X-ray Scattering (GIXS) measurements which are shown in Fig. 4. The width of (h00) crystalline peaks decreases upon thermal and melt annealing in all samples spin-coated from neat solution or with additives and is illustrated in Fig. S1b in ESI. $\dagger$ Relative degree of crystallinity estimated from 

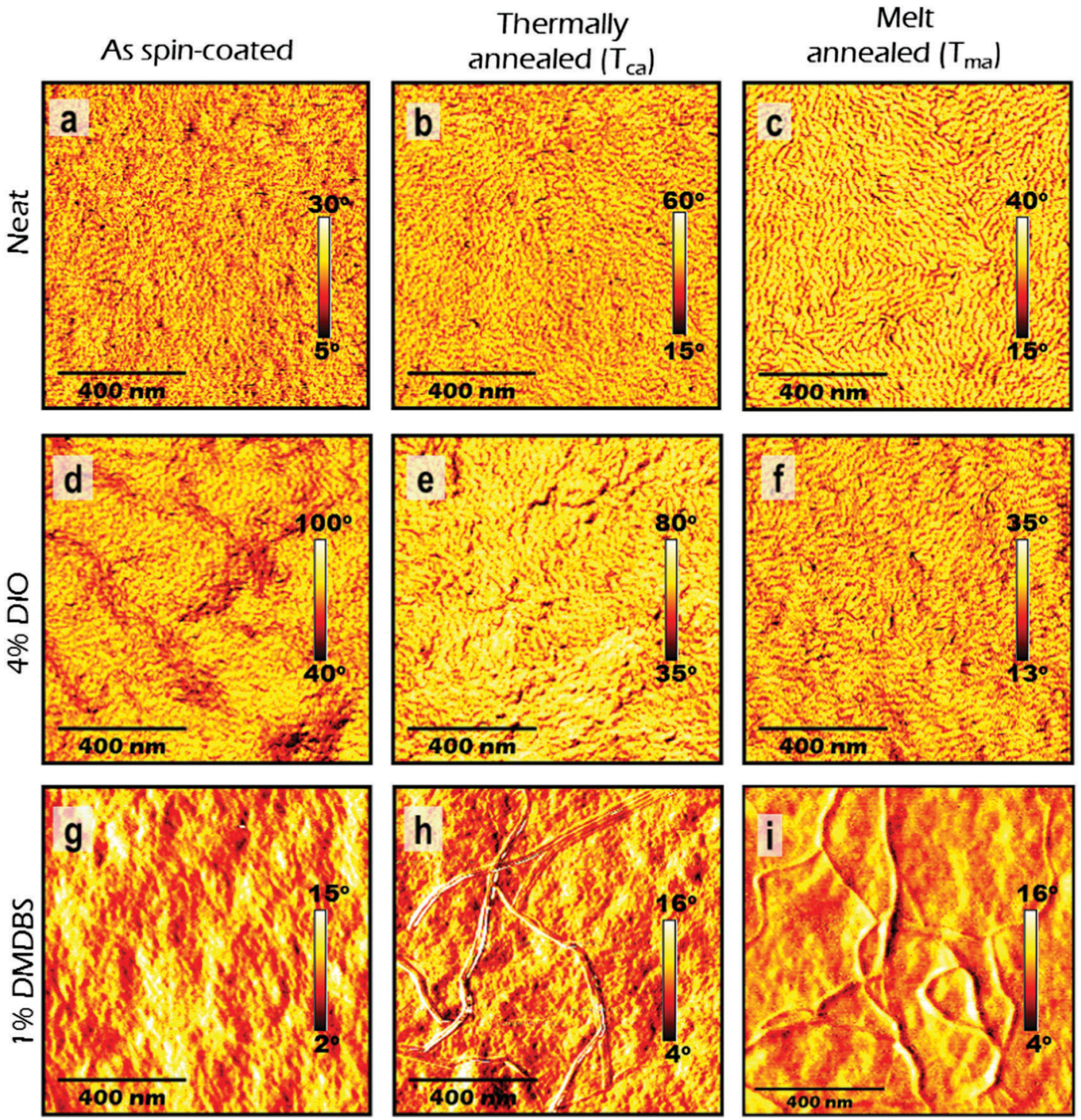

Fig. 3 Atomic force microscopy (AFM) phase images of $100 \mathrm{~nm}$ thick films of P3HT-co-P3DDT spin-coated from neat solution in chlorobenzene (a-c) and with additives of DIO $(\mathrm{d}-\mathrm{f})$ and DMDBS $(\mathrm{g}-\mathrm{i})$. The images in the left column correspond to as-cast films, middle column to thermally annealed films at $T_{\text {ca }}=160{ }^{\circ} \mathrm{C}$ and right column to melt annealed films at $T_{\text {ma }}=200{ }^{\circ} \mathrm{C}$. Scale bar in each image corresponds to $400 \mathrm{~nm}$.

integrated (h00) peak intensity shows that crystallinity increases for each sample with annealing (see Fig. S1 in ESI $\dagger$ for details). Melt annealing increases the crystallinity more than thermal annealing. In comparison to the as spin-coated film of P3HT-co-P3DDT, melt annealing led to nearly a doubling of the crystallite size to $16.5 \mathrm{~nm}$ of the polymer film and an enhancement of rDoC of about $50 \%$ (Fig. $4 \mathrm{c}$ and 3rd row of Table 1$)$. Thermal annealing $\left(T_{\text {ca }}\right)$ gave only a very small effect on the enhancement of $\operatorname{rDoC}(c a .<10 \%$, i.e., within error range) and crystallite sizes (Fig. $4 \mathrm{~b}$ and 2 nd row of Table 1) in comparison to that by melt annealing. Such enhanced crystallinity upon thermal and melt annealing was reported earlier for P3HT. ${ }^{7,26}$ Details about X-ray data analysis are explained in the ESI $\dagger$ (Fig. S1).

\subsection{Influence of solvent vapour annealing}

The influence of controlled solvent $\left(\mathrm{CS}_{2}\right)$ vapour annealing (SvA, see detailed protocol in Experimental section) on the rate constant of exciton-exciton annihilation and degree of crystallinity was explored. The biggest change was observed for $30 \mathrm{~nm}$ thick films. The exciton diffusion coefficient increased more than three times upon solvent vapour annealing and is found to be almost twice bigger than in the melt-annealed film. In general, the process of solvent vapour annealing (SvA) is similar to melt annealing, where solvent swells the film allowing polymer rearrangement around ambient temperature. ${ }^{22,24,42}$ Annealing below or around ambient temperature makes this technique attractive, since it eliminates any possible adverse effect (such as excitonic trap formation, uncontrolled film dewetting, polymer degradation) that may happen at high temperature. Additionally, one could exploit solvent-polymer interaction on the molecular level. ${ }^{22,24,42}$

AFM phase imaging clearly shows extended and prominent lamellar chain orientation in comparison to as spin-coated 

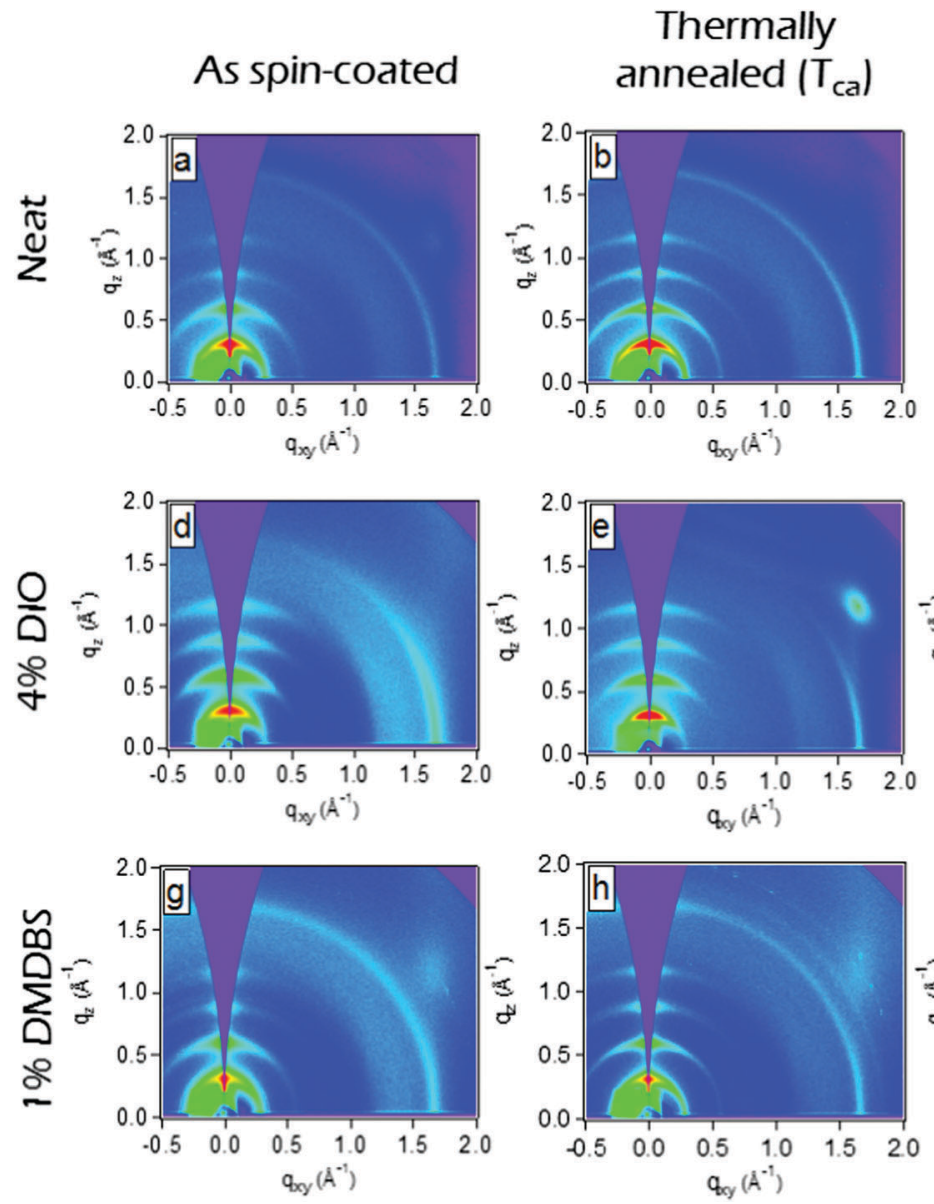
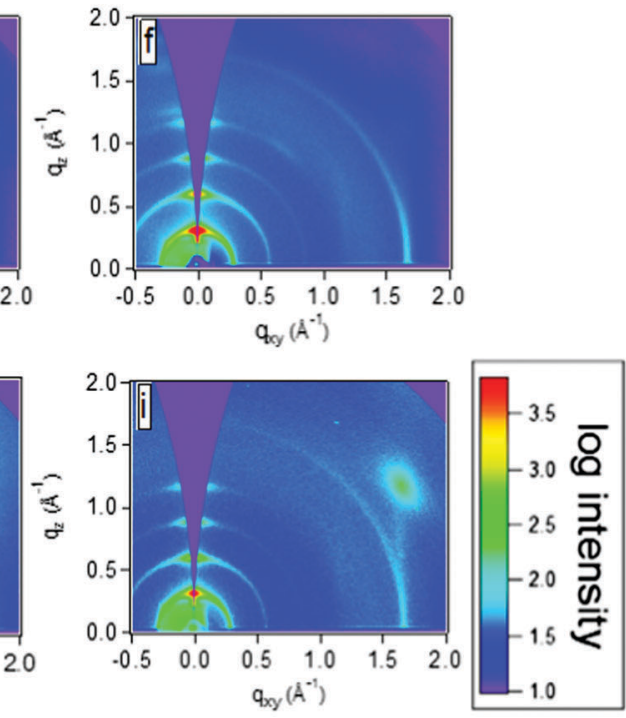

Fig. 4 Grazing incidence X-ray scattering (GIXS) $q_{z}-q_{x y}$ area images of $100 \mathrm{~nm}$ thick films of P3HT-co-P3DDT spin-coated from neat solution in chlorobenzene $(\mathrm{a}-\mathrm{c})$ and with additives of DIO $(\mathrm{d}-\mathrm{f})$ and DMDBS $(\mathrm{g}-\mathrm{i})$. The images in the left column correspond to as-cast films, middle column to thermally annealed films at $T_{\mathrm{ca}}=160{ }^{\circ} \mathrm{C}$ and right column to melt annealed films at $T_{\mathrm{ma}}=200{ }^{\circ} \mathrm{C}$. A decrease of the width of ( $h 00$ ) crystalline peaks is evident in thermally and melt-annealed films which indicates increase of crystallite size. The bright light blue spot in (e) and (i) images are artifacts due to diffuse scattering by Si substrate.

Table 1 Relative degree of crystallinity ( $\mathrm{rDoC}$ ), crystallite size, aggregate fraction $\left(f_{\text {aggr }}\right.$ ), exciton bandwidth $(W)$ and exciton diffusion coefficient $(D)$ values in P3HT-CO-P3DDT films upon varying processing protocols with or without solvent additives and nucleating agent. Top three rows show data for films spin-coated from neat solution in chlorobenzene which were $\sim 100 \mathrm{~nm}$ thick: unprocessed (as spin-coated), thermally annealed at $T_{\mathrm{ca}}=160{ }^{\circ} \mathrm{C}$ (Neat_ $T_{\text {ca }}$ ) and melt annealed at $T_{\text {ma }}=200{ }^{\circ} \mathrm{C}$ (Neat_ $T_{\text {ma }}$ ). The next two rows are for thin $30 \mathrm{~nm}$ films unprocessed (Neat_thin as spin-coated) and solvent vapour annealed (Neat_thin_SvA). Rows 6-8 are films with additive DIO and rows $9-11$ are the films with nucleating agent DMDBS $\left(T_{\text {ca }}=160{ }^{\circ} \mathrm{C}\right.$, $T_{\mathrm{ma}}=200^{\circ} \mathrm{C}$ ). Also, all the films with additive or nucleating agent were casted from solution in chlorobenzene generating film thickness of $\sim 100 \mathrm{~nm}$. Statistical uncertainties in $D$ and $W$ values are up to $\pm 10 \%$. The absolute errors for rDoC are $\pm 10 \%$

\begin{tabular}{|c|c|c|c|c|c|c|}
\hline Sample name & $\operatorname{rDoC}(100)$ & $\operatorname{rDoC}(200)$ & Crystallite size $(\mathrm{nm})$ & $f_{\text {aggr. }}$ & $W(\mathrm{eV})$ & $D \times 10^{-3}\left(\mathrm{~cm}^{2} \mathrm{~s}^{-1}\right)$ \\
\hline Neat_ $T_{\text {ca }}$ & 0.67 & 0.65 & $14.2 \pm 0.9$ & 0.76 & 0.061 & $1.2 \pm 0.1$ \\
\hline Neat_ $T_{\mathrm{ma}}$ & 1 & 1 & $16.5 \pm 0.8$ & 0.76 & 0.079 & $2.1 \pm 0.2$ \\
\hline Neat_thin_SvA & 0.88 & 0.90 & $9.7 \pm 0.9$ & 0.79 & 0.013 & $3.9 \pm 0.4$ \\
\hline$+\mathrm{DIO}$ as spin-coated & 0.76 & 0.65 & $10.1 \pm 0.9$ & 0.76 & 0.062 & $0.7 \pm 0.07$ \\
\hline +DIO_ $T_{\mathrm{ca}}$ & 0.79 & 0.76 & $12.9 \pm 0.7$ & 0.75 & 0.028 & $1.8 \pm 0.2$ \\
\hline+ DMDBS_ $T_{\mathrm{ca}}$ & 0.79 & 0.82 & $13 \pm 1$ & 0.77 & 0.092 & $1.6 \pm 0.2$ \\
\hline +DMDBS_ $T_{\mathrm{ma}}$ & 0.86 & 0.91 & $17.2 \pm 0.7$ & 0.77 & 0.068 & $1.5 \pm 0.2$ \\
\hline
\end{tabular}

film (Fig. 5a and b). Further investigation by GIXS shows an increase in $\mathrm{rDoC}$ in comparison to the neat as spin-coated thin film (Fig. $5 \mathrm{c}$ and $\mathrm{d}$ and $4-5$ th rows of Table 1). We note that SvA experiment was done in a thin film of $c a .30 \mathrm{~nm}$ and also compared with as spin-coated film of similar thickness made of neat polymer. The thinner as-cast film has slightly lower rDoC, 

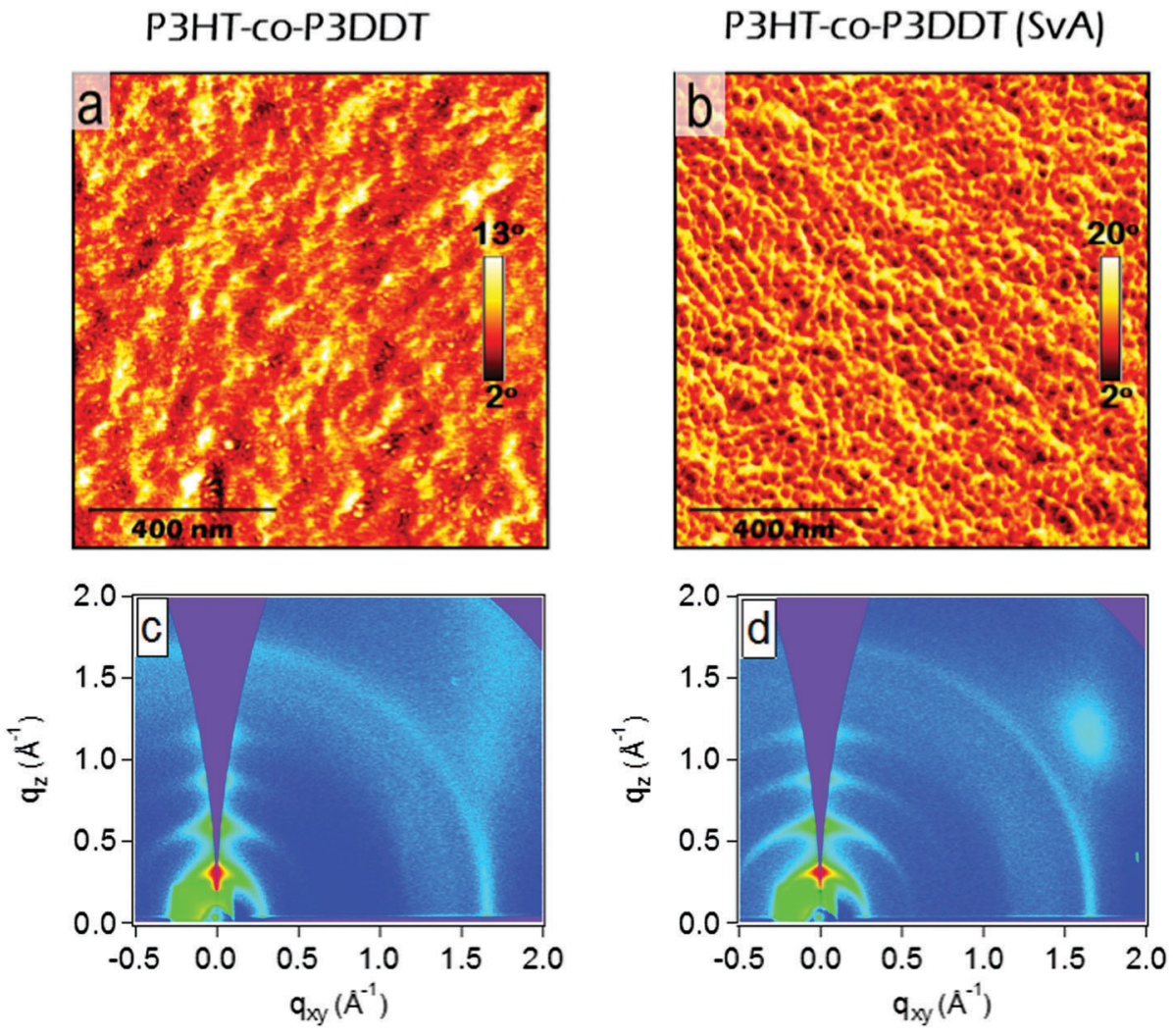

Fig. 5 AFM phase images ( $a$ and b) and GIXS $q_{z}-q_{x y}$ area images ( $c$ and d) showing $30 \mathrm{~nm}$ thick P3HT-co-P3DDT films spin-coated from neat solution in chlorobenzene before (a) and after (b) solvent $\left(\mathrm{CS}_{2}\right)$ annealing. Solvent vapour annealing lead enhancement in relative degree of crystallinity, accompanied with notably extended and oriented lamellar structures as shown in AFM phase image. Scale bars in each AFM image correspond to $400 \mathrm{~nm}$.

which was also observed in poly(3-ethylhexylthiophene) (P3EHT). ${ }^{35}$ However, the exciton diffusion coefficient was found to be the same for $30 \mathrm{~nm}$ and $100 \mathrm{~nm}$ thick as cast films.

\subsection{Influence of processing additives}

We found that the singlet exciton diffusion coefficient decreased when films were spin-coated from solutions with additives DIO or DMDBS (see Fig. 2 and Table 1 for details). However, when films spin-coated with these additives were thermally annealed, the exciton diffusion coefficient was higher by $30-50 \%$ as compared to films spin-coated from neat solutions. This shows that a combination of additives with thermal annealing at a temperature just above the crystallisation temperature can be advantageous for preparing organic solar cells using polythiophenes as electron donors because the excitons can reach an interface with an electron acceptor quicker and generate charge carriers more efficiently. AFM height topography shows structured film surfaces with higher roughness (Fig. S6d-g, ESI $\dagger$ ). This may be due to trapped traces of $\mathrm{DIO}^{43,44}$ (even though the samples were kept at $10^{-5}$ mbar vacuum for 4-5 hours). Interestingly, when such films were thermally or melt annealed their roughness got significantly reduced (Fig. S6d-f, ESI $\dagger$ ). AFM phase imaging in films made from polymer solution containing DIO show increase in the length of lamellae upon thermal or melt annealing in a similar fashion to films without it. In the case of films made from polymer solution containing DMDBS $(1 \% \mathrm{w} / \mathrm{w})$ there are notable microfibrillar features (Fig. 3h, i and Fig. S6h, i, ESI $\dagger$ ), which may be due to the crystallisation of the DMDBS itself and are particularly prominent upon thermal or melt annealing..$^{20,21,45}$ Phase contrast images in DMDBS added films do not show any notable lamellar structures from the polymer (see Fig. $3 g$ and i). This is probably due to the formation of mixed polymer-nucleating agent interface.

All films prepared using the solvent additives show a slight increase in rDoC in comparison to the film spin-coated from neat solutions. We observe a $12 \%$ increase of rDoC in as spincoated film with the addition of DMDBS whilst the crystallite size was comparable within error. When films were melt-annealed, a bigger enhancement of rDoC was observed in films without DMDBS which suggests that DMDBS gives no advantage when films are melt-annealed. A similar observation has been reported earlier for P3DDT, where no enhancement in the heat of fusion was observed in films processed with DMDBS. ${ }^{20}$ This correlates with smaller increase of exciton diffusivity in films prepared with additives upon melt annealing as compared to the films spincoated from neat solution.

\section{Discussion}

In this section we consider what structural and spectral parameters influence exciton diffusion. Fig. 6a shows normalized absorption spectra of various differently processed P3HT-co-P3DDT films. 

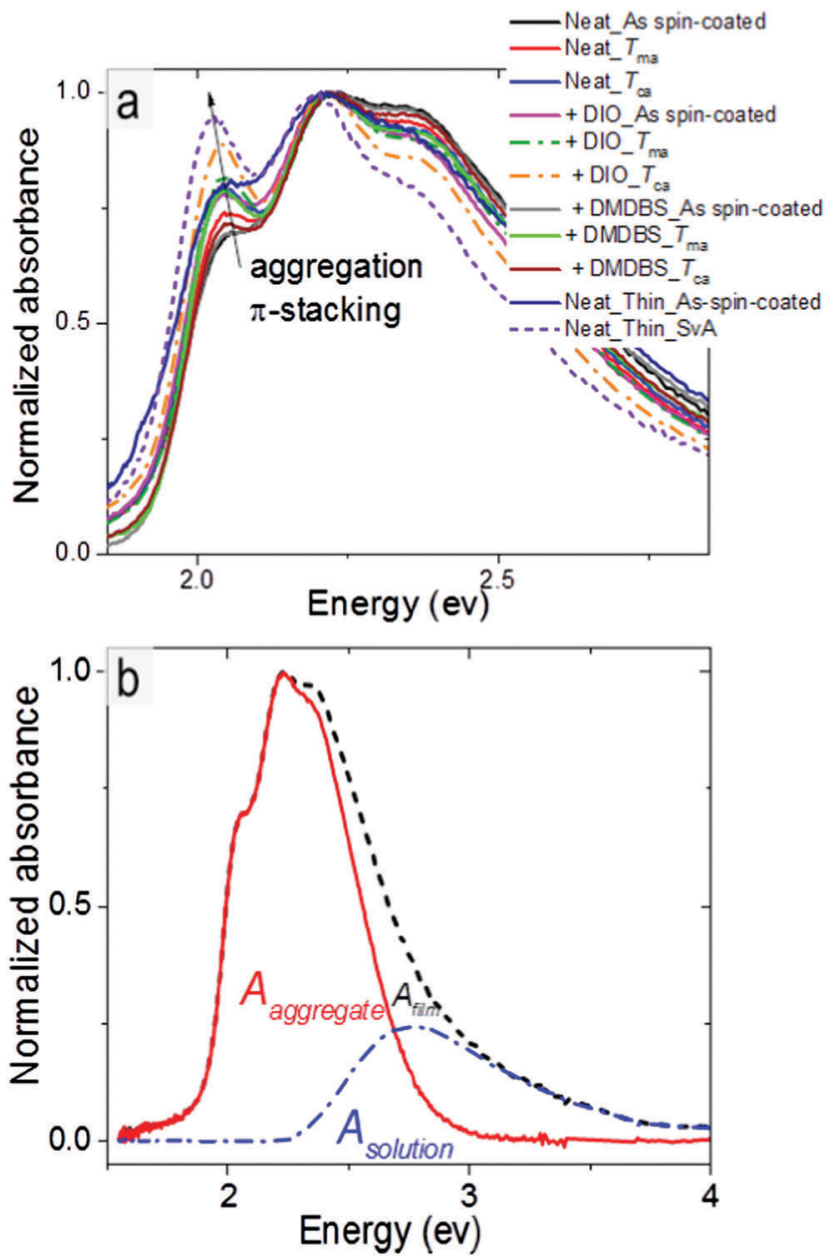

Fig. 6 (a) Normalised absorption spectra of P3HT-co-P3DDT films spincoated from neat solution and with additives annealed under different conditions. (b) Representative absorption spectra of P3HT-co-P3DDT in film (dashed black line) and solution (blue line). The aggregate absorption spectrum (red line) is found by subtracting the solution spectrum from the film spectrum.

All films show a shoulder at $2.05 \mathrm{eV}\left(A_{0-0}\right)$ and a peak at $2.23 \mathrm{eV}$ $\left(A_{0-1}\right)$. The peak at $2.05 \mathrm{eV}$ increases with annealing and also after addition of DIO and suggests an increase of conjugation length because of aggregation and $\pi-\pi$ stacking of the conjugated chains. To determine the absorption spectrum of the aggregated chromophores in films we used their absorption spectra and subtracted the contribution of the amorphous phase which we assumed to be identical to the solution absorption as shown in Fig. $6 b^{32}$ By integrating the absorption spectrum of the aggregated chromophores over photon energy and dividing it by the integral of the total absorption spectrum over photon energy we determined the aggregate fraction in different films $\left(f_{\text {aggr. }}\right)$ which are given in Table 1 . The values of $f_{\text {aggr. }}$ vary over a small range between 0.75 and 0.79 and show a weak correlation with rDoC. This observation is similar to that reported in a different polythiophene where no clear dependence of rDoC on the aggregate fraction has been observed ${ }^{35}$ This is not surprising because rDoC is mainly determined by the alkyl stacking along the (h00) direction, whereas aggregation is mainly influenced by $\pi-\pi$ stacking (Fig. 1d).

We estimated the free exciton bandwidth $W$ from the shape of the absorption spectra of aggregated chromophores using the weakly-interacting $\mathrm{H}$-aggregate model developed by Spano et $a{ }^{46}$ When a Huang-Rhys factor for vibronic coupling of isolated chain is 1 , then the ratio of the $A_{0-0}$ and $A_{0-1}$ absorption peaks of aggregated chains

$$
\frac{A_{0-0}}{A_{0-1}} \approx\left(\frac{1-\frac{0.24 W}{E_{\mathrm{p}}}}{1+\frac{0.073 W}{E_{\mathrm{p}}}}\right)^{2}
$$

where $E_{\mathrm{p}}=0.18 \mathrm{eV}$ is an apparent energy of the main vibration coupled to absorption. ${ }^{32,46}$

This ratio was determined by fitting the absorption spectrum of the aggregated fraction with Gaussian functions as described in ESI. $\dagger$ The obtained $W$ values are tabulated in Table 1. Smaller $W$ indicates a longer conjugation length which also promotes exciton delocalisation along the conjugated chain. ${ }^{32,46}$ Our results show smaller $W$ in films fabricated with DIO additive which is consistent with previous observation. ${ }^{18}$ The smallest $W$ is observed in the solvent vapour annealed thin $30 \mathrm{~nm}$ film and implies the longest conjugation of the aggregated polymer chains. ${ }^{46}$ Using the same annealing conditions on a $100 \mathrm{~nm}$ film did not induce any change in the absorption spectrum suggesting no notable change in local chain order and conjugation length. It may be possible to induce structural changes in $100 \mathrm{~nm}$ films by further adjusting the annealing temperature, flow rate and vapour pressure.

The correlation plots of exciton diffusivity $D$ with the relative degree of crystallinity along alkyl-stacking $\operatorname{rDoC}(100)$ and exciton bandwidth $W$ are shown in Fig. 7a-c. Both rDoC and $W$ change by applying different processing protocols. The general trend is that a higher $\operatorname{rDoC}(100)$ and lower $W$ result in higher exciton diffusivity. The rDoC is comparable in solvent-vapour annealed film and melt-annealed films (within experimental error). However, the $W$ is approximately six times lower in solvent-vapour annealed film indicating a longer conjugation of aggregated chains in this film. Higher exciton diffusivity in solvent-vapour annealed film can be explained by longer conjugation. The dependence of $D$ on rDoC(100) is relatively weak, a better correlation is observed between $D$ and the ratio of $\operatorname{rDoC}(100)$ to $W$. Exciton diffusivity shows a weak correlation with the crystallite size, including the solvent-vapour annealed film which shows high diffusivity and moderate crystallite size (Fig. S5, ESI $\dagger$ ), hence crystallite size is not a decisive parameter.

In our experiments we found that films prepared with solvent additives (DIO, DMDBS) and thermally annealed at a temperature just above the crystallisation temperature increased exciton diffusivity by $30-50 \%$ as compared to films spin-coated from neat solutions. This indicates that a combination of additives with thermal annealing can be advantageous for preparing organic solar cells using polythiophenes as electron donors because the excitons can reach an interface with an electron acceptor quicker and generate charge carriers more efficiently. 

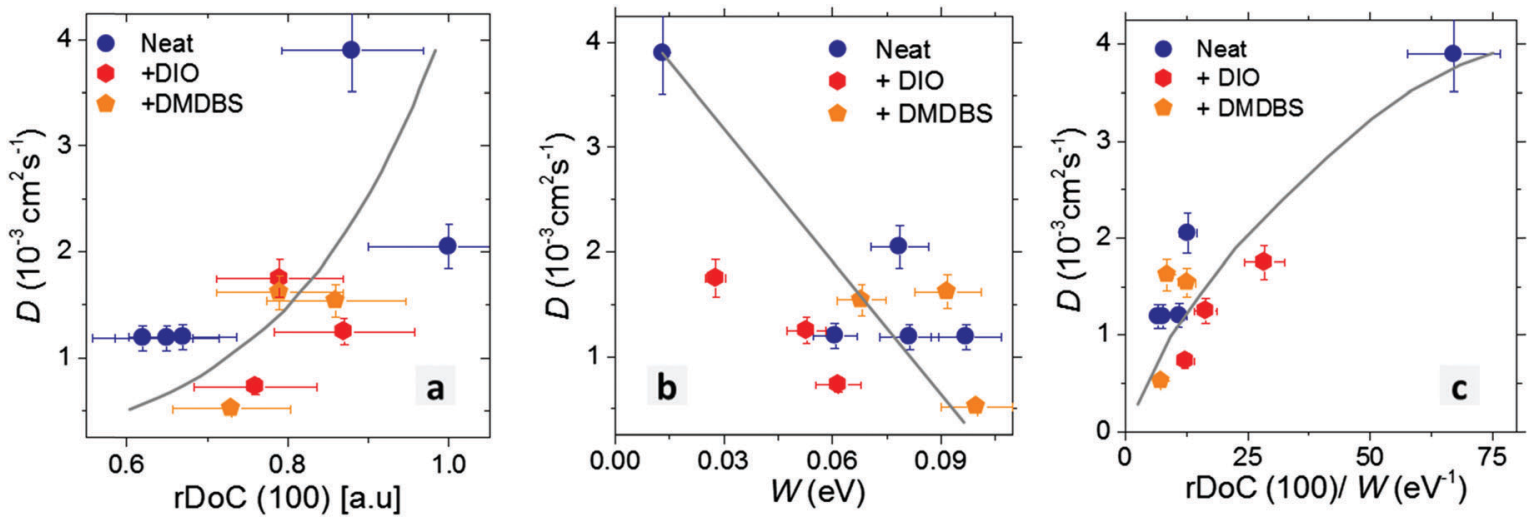

Fig. 7 Dependence of exciton diffusion coefficient $D$ in P3HT-co-P3DDT films on a relative degree of crystallinity rDoC(100) (a); free exciton bandwidth $W$ (b); and on the ratio of $\mathrm{rDoC}(100)$ and $W$ (c). Films were spin-coated from neat solutions or with additives as indicated in the legend. Data points at $D=3.9 \times 10^{-3} \mathrm{~cm}^{2} \mathrm{~s}^{-1}$ which give one-dimensional diffusion length of $10 \mathrm{~nm}$ correspond to the solvent-vapour annealed thin film. Solid lines are guides to eye only.

\section{Conclusions}

In this work we have demonstrated that control of processing can strongly influence exciton diffusion, degree of crystallinity and exciton bandwidth. We found that melt annealing leads to $\sim 50 \%$ enhancement in both degree of crystallinity and exciton diffusion coefficient in films of copolymer P3HT-co-P3DDT. Controlled solvent vapour annealing has the strongest effect on exciton diffusion coefficient leading to an enhancement by more than a factor of three. The results show that melt annealing and solvent vapour annealing are effective ways to enhance exciton diffusivity. A combination of solvent additives (DIO, DMDBS) with thermal annealing at a temperature just above the crystallisation temperature increased exciton diffusivity by $30-50 \%$ as compared to films spincoated from neat solutions. We show that the enhancement of exciton diffusivity is a combined result of increased crystallinity of alkyl-stacking and longer conjugation of aggregated chains which reduces the exciton bandwidth. We expect a similar trend in other semi-crystalline conjugated polymers with alkyl- and $\pi$-stacking, suggesting that developing the materials and processing methods that enhance crystallinity and conjugation length is a useful approach to increasing exciton diffusion.

\section{Experimental section}

\subsection{Materials and characterisation}

We synthesized a P3HT-co-P3DDT using a Kumada catalyst transfer copolymerisation reaction involving LiCl promoted Grignard monomer activation. ${ }^{47,48}$ Protocols of synthesis and characterisations could be found in ESI. $\dagger$ 1,8-Diiodooctane (DIO) was purchased from Sigma-Aldrich. It was added as it is (as liquid) into the polymer solution $(4 \% \mathrm{v} / \mathrm{v})$ before spin-coating. Polymer nucleating agent 1,3:2,4-di(3,4-dimethylbenzylidene)sorbitol (DMDBS) was purchased from Chemos Gmbh and further used as it is (as solid powder) (1\% w/w) into the solid polymer. The mixture was further dissolved in chlorobenzene, kept at $50{ }^{\circ} \mathrm{C}$ overnight while stirring, then filtered by $0.1 \mu \mathrm{m}$ pore filter before spin-coating.

\subsection{Film preparation and annealing protocols}

Polymer was dissolved in chlorobenzene by stirring overnight at $50{ }^{\circ} \mathrm{C}$ in a $\mathrm{N}_{2}$-filled glove box. Fused silica substrates were cleaned via ultrasonication for 15 minutes first with acetone and then with isopropanol. The films were spin-coated in the glove box $\left(\mathrm{O}_{2}\right.$ level less than $\left.0.5 \mathrm{ppm}\right)$ using spin speeds in the range of 1400-1600 rpm. All the spin-coated films in our studies have thickness of $c a .100 \mathrm{~nm}$, except ultrathin films of $c a .30 \mathrm{~nm}$ used for solvent vapour annealing and thickness comparison.

We explored two different temperatures for annealing, thereby exploring the effect of different amounts of polymer chain reorganisation. Our annealing temperatures were a few degrees above crystallisation temperature by thermal annealing $\left(T_{\mathrm{ca}}=160^{\circ} \mathrm{C}\right)$ and few degrees above melting point by melt annealing $\left(T_{\text {ma }}=200{ }^{\circ} \mathrm{C}\right)$ respectively. To further recrystallize mobile polymer chains from more mobile state or melt, we did slow controlled cooling towards room temperature. Both the thermal and melt annealing were done through series of controlled heating and cooling protocols inside a Linkam microscope hot stage under continuous flow of nitrogen. Occasional monitoring through the optical microscope ensured the quality of the annealing by avoiding any defects such as dewetting. ${ }^{42}$ Thermal annealing $\left(T_{\text {ca }}\right)$ : heating at $90{ }^{\circ} \mathrm{C} \min ^{-1}$ from room temperature to $160{ }^{\circ} \mathrm{C}$, cooling at $2{ }^{\circ} \mathrm{C} \mathrm{min} \min ^{-1}$ to $157{ }^{\circ} \mathrm{C}$, holding $5 \mathrm{~min}$ at $157{ }^{\circ} \mathrm{C}$, cooling at $1{ }^{\circ} \mathrm{C} \mathrm{m^{-1 }}$ to room temperature. Melt annealing ( $\left.T_{\text {ma }}\right)$ : heating at $90{ }^{\circ} \mathrm{C} \mathrm{min}{ }^{-1}$ from room temperature to $200{ }^{\circ} \mathrm{C}$, holding $8 \mathrm{~min}$ at $200{ }^{\circ} \mathrm{C}$, cooling at $4{ }^{\circ} \mathrm{C} \min ^{-1}$ to $170{ }^{\circ} \mathrm{C}$, cooling at $1{ }^{\circ} \mathrm{C} \mathrm{min}^{-1}$ to room temperature.

We used solvent vapour annealing on thin $(c a .30 \mathrm{~nm}) \mathrm{spin}-$ coated film of the neat polymer by controlling the flow of carbon disulphide $\left(\mathrm{CS}_{2}\right)$ vapour into a custom-built solvent vapour annealing chamber, which was placed under an optical microscope for monitoring the process in situ. We exposed spin-coated ultrathin solid films to solvent vapour by placing the thin film on a Peltier element enclosed inside the sample chamber. The chamber is capable of flushing with nitrogen saturated with solvent vapour. The Peltier element allowed us to control the sample temperature and thus the amount of solvent which could 
condense onto a cold film. Before the experiment, the sample chamber was separately flushed with pure nitrogen for $2 \mathrm{~min}$ to remove any moisture or unwanted gas. Further a mass flow controller was used at $25 \mathrm{sccm}$ flow to expose as spin-coated polymer films to control amounts of nitrogen saturated with a $\mathrm{CS}_{2}$ solvent vapour. During the whole experiment, nitrogen gas was bubbling through the bottle of solvent which was kept at constant temperature of $46{ }^{\circ} \mathrm{C}$ (boiling point of $\mathrm{CS}_{2}$ ), by placing it inside a water bath. Such flow of solvent was allowed for 3 min to make the chamber nearly full with solvent vapour, before turning on the Peltier element for cooling. Further sample was cooled at $c a .7-8{ }^{\circ} \mathrm{C}$ temperature for $1 \mathrm{~min}$ while the solvent flow is still on. After switching off the cooling, the sample was allowed to heat up to ambient temperature slowly. The film was dried under dry nitrogen gas flow, keeping inside the sample chamber. The whole process was monitored under optical microscope through quartz window on the top lid of sample chamber to ensure the quality of the annealing (monitoring changes during polymer swelling; avoiding dewetting and ruptures).

\subsection{Measurements on films}

Grazing incidence X-ray scattering (GIXS). Films for X-ray scattering experiments are fabricated by spin-coating P3HT-coP3DDT solutions onto $\mathrm{Si}(100)$ wafers, in a similar way as done on fused silica substrates for photophysical studies. Diffraction patterns are collected using an incident beam of $12.7 \mathrm{keV}$ (at BL11-3, SLAC, CA, USA) at a grazing angle of $0.12^{\circ}$ and expressed as a function of the scattering vector $q=4 \pi \sin (\theta) / \lambda$. Here, $\theta$ represents half of the scattering angle, $\lambda$ is the wavelength of the incident beam, $q_{x y}$ is the component of the scattering vector parallel to the substrate plane and $q_{z}$ is the component perpendicular to the substrate plane. 2D-diffraction images were collected on MAR 345 image plate at $400 \mathrm{~mm}$ distance, further analysed either by WxDiff, ${ }^{36}$ WAXStools $^{49}$ and Nika. ${ }^{50}$ Details of further analysis including $\mathrm{rDoC}$ and crystallite size are explained in $\mathrm{ESI} \dagger$ (Fig. S1).

Atomic force microscopy (AFM). Topographical morphology and phase imaging was performed on Bruker MultiMode 8 atomic force microscope in the tapping mode. NANOSENSORS ${ }^{\mathrm{TM}}$ PPPNCSTR tips with force constant of $6-7 \mathrm{~N} \mathrm{~m}^{-1}$ were used as AFM probes.

Ellipsometry. Film thicknesses were measured with a variable angle spectroscopic ellipsometer Woolam WVASE ${ }^{\circledR}$ and also estimated from a Veeco Dektak 150 surface profiler. Film thicknesses used in exciton-exciton annihilation measurements varied between $c a .30$ to $c a .100 \mathrm{~nm}$.

Absorption spectra. The absorbance spectra of the films were measured using a Varian Cary 300 UV-Vis Spectrophotometer.

Exciton-exciton annihilation. Fluorescence decays at low excitation density were measured using 100 fs pulses with $80 \mathrm{MHz}$ repetition rate at $400 \mathrm{~nm}$ for excitation and a Hamamatsu C6860 synchroscan streak camera for detection. For exciton-exciton annihilation measurements using fluorescence the excitation was with $100 \mathrm{fs}$ pulses at $5 \mathrm{kHz}$ repetition rate at $400 \mathrm{~nm}$. All fluorescence measurements were performed under a vacuum of $10^{-5}$ mbar. The energy of the laser pulses was controlled with neutral density filters. Excitation spot sizes were measured with a LaserCam 3D beam profiler.

\section{Acknowledgements}

M. C., M. T. S., A. R. and I. D. W. S. acknowledge support from the European Research Council (EXCITON grant 321305). I. D. W. S. acknowledges Royal Society Wolfson Research Merit Award. Use of the Stanford Synchrotron Radiation Lightsource, SLAC National Accelerator Laboratory, is supported by the U.S. Department of Energy, Office of Science, Office of Basic Energy Sciences under Contract No. DE-AC02-76SF00515. The research data supporting this publication can be accessed at http:// dx.doi.org/10.17630/9e0ae16c-0033-46c8-a536-52c51b136d24.

\section{References}

1 J. Zhao, Y. Li, G. Yang, K. Jiang, H. Lin, H. Ade, W. Ma and H. Yan, Nat. Energy, 2016, 1, 15027.

2 M. A. Baklar, F. Koch, A. Kumar, E. B. Domingo, M. CampoyQuiles, K. Feldman, L. Yu, P. Wobkenberg, J. Ball, R. M. Wilson, I. McCulloch, T. Kreouzis, M. Heeney, T. Anthopoulos, P. Smit and N. Stingelin, Adv. Mater., 2010, 22, 3942-3947.

3 W. Ma, G. Yang, K. Jiang, J. H. Carpenter, Y. Wu, X. Meng, T. McAfee, J. Zhao, C. Zhu, C. Wang, H. Ade and Y. He, Adv. Energy Mater., 2015, 5, 23.

4 G. J. Hedley, A. J. Ward, A. Alekseev, C. T. Howells, E. R. Martins, L. A. Serrano, G. Cooke, A. Ruseckas and I. D. Samuel, Nat. Commun., 2013, 4, 2867.

5 B. P. Rand, C. Girotto, A. Mityashin, A. Hadipour, J. Genoe and P. Heremans, Appl. Phys. Lett., 2009, 95, 173304.

6 S. M. Menke, W. A. Luhman and R. J. Holmes, Nat. Mater., 2013, 12, 152-157.

7 M. Sim, J. Shin, C. Shim, M. Kim, S. B. Jo, J.-H. Kim and K. Cho, J. Phys. Chem. C, 2014, 118, 760-766.

8 H. Najafov, B. Lee, Q. Zhou, L. Feldman and V. Podzorov, Nat. Mater., 2010, 9, 938-943.

9 R. R. Lunt, N. C. Giebink, A. A. Belak, J. B. Benziger and S. R. Forrest, J. Appl. Phys., 2009, 105, 053711.

10 O. V. Mikhnenko, J. Lin, Y. Shu, J. E. Anthony, P. W. Blom, T.-Q. Nguyen and M. A. Loi, Phys. Chem. Chem. Phys., 2012, 14, 14196-14201.

11 M. T. Sajjad, A. J. Ward, C. Kaestner, A. Ruseckas, H. Hoppe and I. D. Samuel, J. Phys. Chem. Lett., 2015, 6, 3054-3060.

12 A. L. Ayzner, J. Mei, A. Appleton, D. DeLongchamp, A. Nardes, S. Benight, N. Kopidakis, M. F. Toney and Z. Bao, ACS Appl. Mater. Interfaces, 2015, 7, 28035-28041.

13 J. E. Kroeze, T. J. Savenije, M. J. Vermeulen and J. M. Warman, J. Phys. Chem. B, 2003, 107, 7696-7705.

14 G. Dicker, M. P. de Haas, L. D. Siebbeles and J. M. Warman, Phys. Rev. B: Condens. Matter Mater. Phys., 2004, 70, 045203.

15 S. Holliday, R. S. Ashraf, A. Wadsworth, D. Baran, S. A. Yousaf, C. B. Nielsen, C.-H. Tan, S. D. Dimitrov, Z. Shang, N. Gasparini, M. Alamoudi, F. Laquai, C. J. Brabec, A. Salleo, J. R. Durrant and I. McCulloch, Nat. Commun., 2016, 7, 11585. 
16 J. D. Lin, O. V. Mikhnenko, T. S. van der Poll, G. C. Bazan and T.-Q. Nguyen, Adv. Mater., 2015, 27, 2528-2532.

17 F. Liu, Y. Gu, X. Shen, S. Ferdous, H.-W. Wang and T. P. Russell, Prog. Polym. Sci., 2013, 38, 1990-2052.

18 Y. Long, A. J. Ward, A. Ruseckas and I. D. Samuel, Synth. Met., 2016, 216, 23-30.

19 V. Pranculis, A. Ruseckas, D. A. Vithanage, G. J. Hedley, I. D. Samuel and V. Gulbinas, J. Phys. Chem. C, 2016, 120, 9588-9594.

20 N. D. Treat, J. A. N. Malik, O. Reid, L. Yu, C. G. Shuttle, G. Rumbles, C. J. Hawker, M. L. Chabinyc, P. Smith and N. Stingelin, Nat. Mater., 2013, 12, 628-633.

21 A. Sharenko, N. D. Treat, J. A. Love, M. F. Toney, N. Stingelin and T.-Q. Nguyen, J. Mater. Chem. A, 2014, 2, 15717-15721.

22 E. J. Crossland, K. Rahimi, G. Reiter, U. Steiner and S. Ludwigs, Adv. Funct. Mater., 2011, 21, 518-524.

23 N. Basu, A. Osichow, S. Mecking and G. Reiter, Eur. Phys. J. E: Soft Matter Biol. Phys., 2012, 35, 1-12.

24 C. Sinturel, M. Vayer, M. Morris and M. A. Hillmyer, Macromolecules, 2013, 46, 5399-5415.

25 Y. Wang, B. Heck, D. Schiefer, J. O. Agumba, M. Sommer, T. Wen and G. Reiter, ACS Macro Lett., 2014, 3, 881-885.

26 C. R. Singh, G. Gupta, R. Lohwasser, S. Engmann, J. Balko, M. Thelakkat, T. Thurn-Albrecht and H. Hoppe, J. Polym. Sci., Part B: Polym. Phys., 2013, 51, 943-951.

27 O. Mikhnenko, F. Cordella, A. Sieval, J. Hummelen, P. Blom and M. Loi, J. Phys. Chem. B, 2008, 112, 11601-11604.

28 I. Botiz and N. Stingelin, Materials, 2014, 7, 2273-2300.

29 K. Zhao, H. U. Khan, R. Li, Y. Su and A. Amassian, Adv. Funct. Mater., 2013, 23, 6024-6035.

30 F. P. V. Koch, J. Rivnay, S. Foster, C. Muellerller, J. M. Downing, E. Buchaca-Domingo, P. Westacott, L. Yu, M. Yuan, M. Baklar, Z. Fei, C. Luscombe, M. A. McLachlan, M. Heeney, G. Rumbles, C. Silva, A. Salleo, J. Nelson, P. Smith and N. Stingelin, Prog. Polym. Sci., 2013, 38, 1978-1989.

31 P. E. Shaw, A. Ruseckas and I. D. Samuel, Adv. Mater., 2008, 20, 3516-3520.

32 Z. Masri, A. Ruseckas, E. V. Emelianova, L. Wang, A. K. Bansal, A. Matheson, H. T. Lemke, M. M. Nielsen, H. Nguyen, O. Coulembier, P. Dubois, D. Beljonne and I. D. Samuel, Adv. Energy Mater., 2013, 3, 1445-1453.
33 Y. Tamai, Y. Matsuura, H. Ohkita, H. Benten and S. Ito, J. Phys. Chem. Lett., 2014, 5, 399-403.

34 J. Rivnay, S. C. Mannsfeld, C. E. Miller, A. Salleo and M. F. Toney, Chem. Rev., 2012, 112, 5488-5519.

35 D. T. Duong, V. Ho, Z. Shang, S. Mollinger, S. C. Mannsfeld, J. Dacuña, M. F. Toney, R. Segalman and A. Salleo, Adv. Funct. Mater., 2014, 24, 4515-4521.

36 L. H. Jimison, A. Salleo, M. McGehee and M. F. Toney, Understanding microstructure and charge transport in semicrystalline polythiophenes, Stanford University, 2011.

37 B. W. Boudouris, V. Ho, L. H. Jimison, M. F. Toney, A. Salleo and R. A. Segalman, Macromolecules, 2011, 44, 6653-6658.

38 S. T. Salammal, S. Dai, U. Pietsch, S. Grigorian, N. Koenen, U. Scherf, N. Kayunkid and M. Brinkmann, Eur. Polym. J., 2015, 67, 199-212.

39 V. Ho, B. W. Boudouris and R. A. Segalman, Macromolecules, 2010, 43, 7895-7899.

40 A. Zen, M. Saphiannikova, D. Neher, U. Asawapirom and U. Scherf, Chem. Mater., 2005, 17, 781-786.

41 P.-T. Wu, G. Ren and S. A. Jenekhe, Macromolecules, 2010, 43, 3306-3313.

42 A. Clough, M. Chowdhury, K. Jahanshahi, G. Reiter and O. K. C. Tsui, Macromolecules, 2012, 45, 6196-6200.

43 L. Zhao, S. Zhao, Z. Xu, Q. Yang, D. Huang and X. Xu, Nanoscale, 2015, 7, 5537-5544.

44 N. Herath, S. Das, J. K. Keum, J. Zhu, R. Kumar, I. N. Ivanov, B. G. Sumpter, J. F. Browning, K. Xiao, G. Gu, P. Joshi, S. Smith and V. Lauter, Sci. Rep., 2015, 5, 13407.

45 J. Lipp, M. Shuster, A. E. Terry and Y. Cohen, Langmuir, 2006, 22, 6398-6402.

46 J. Clark, J.-F. Chang, F. C. Spano, R. H. Friend and C. Silva, Appl. Phys. Lett., 2009, 94, 163306.

47 J.-P. Lamps and J.-M. Catala, Macromolecules, 2011, 44, 7962-7968.

48 S. Wu, L. Huang, H. Tian, Y. Geng and F. Wang, Macromolecules, 2011, 44, 7558-7567.

49 S. D. Oosterhout, V. Savikhin, J. Zhang, Y. Zhang, M. A. Burgers, S. R. Marder, G. C. Bazan and M. F. Toney, Chem. Mater., 2017, 29, 3062-3069.

50 J. Ilavsky, J. Appl. Crystallogr., 2012, 45, 324-328. 